Supporting Information For

\title{
Preparation of Acentric Porous Coordination Frameworks From Inter- penetrated Diamondoid Array Through Anion-Exchange Procedures: Crystal Structures and Properties
}

Miao Du, Ya-Mei Guo, Shen-Tan Chen, Xian-He Bu,* Stuart R. Batten, Joan Ribas and Susumu Kitagawa

Department of Chemistry, Nankai University, Tianjin 300071, China; and School of Chemistry, PO Box 23, Monash University, Clayton, Victoria, 3800, Australia; and Departament de Química Inorgànica, Universitat de Barcelona, Diagonal, 647, 08028-Barcelona, Spain and Department of Synthetic Chemistry and Biological Chemistry, Graduate School of Engineering, Kyoto University, Yoshida, Sakyo-ku, Kyoto 606-8501, Japan 


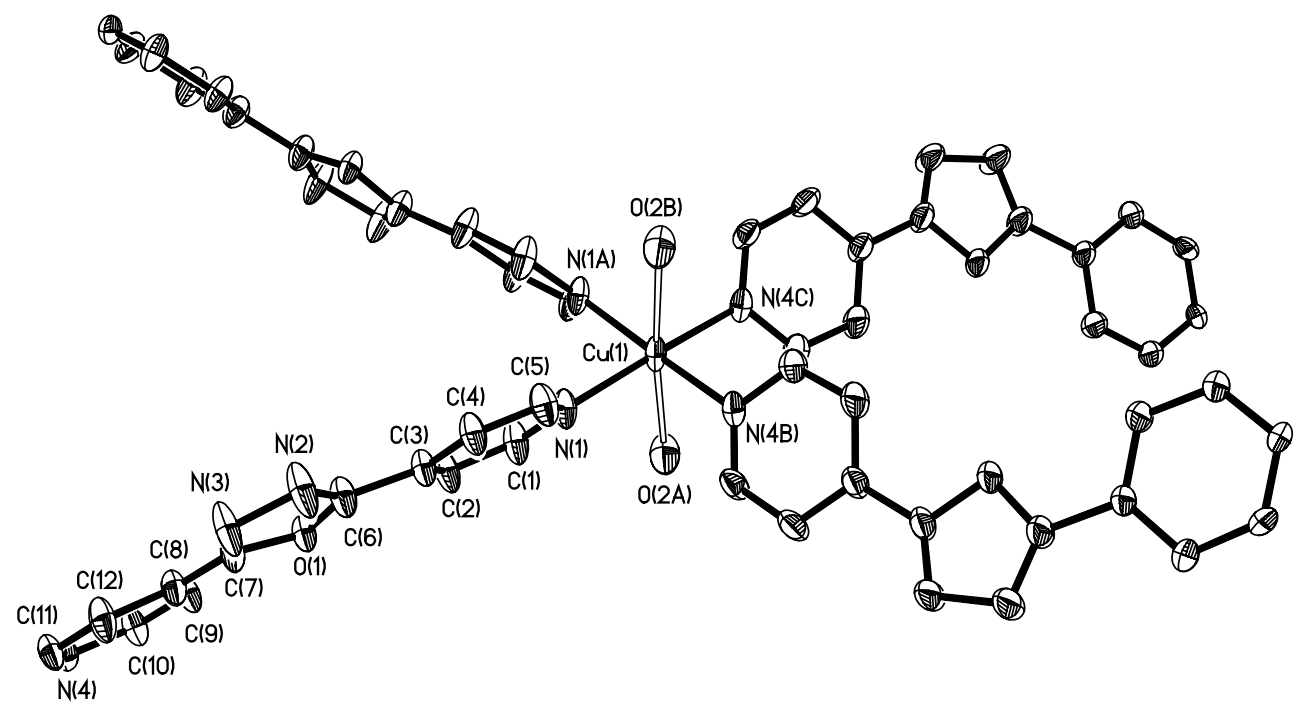

Figure S1. Coordination environment of $\mathrm{Cu}^{\mathrm{II}}$ in complex 1. 


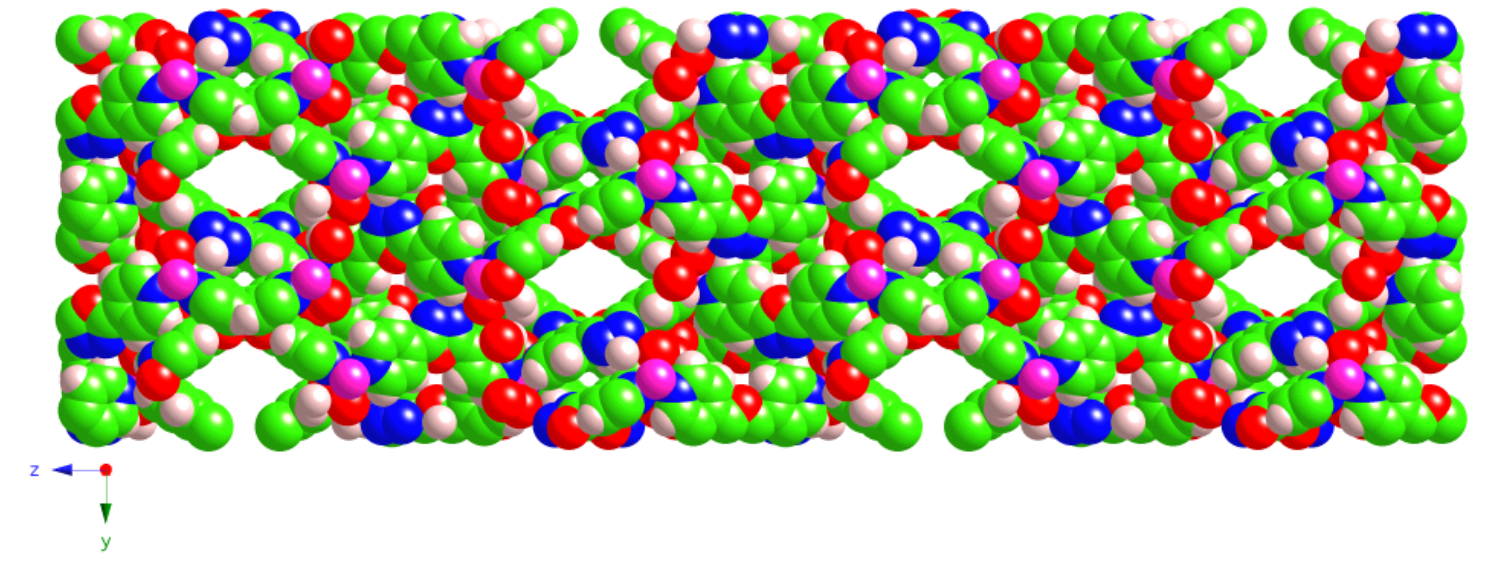

Figure S2. Space-filling model of 1 exhibiting the channels along [100] or [010] direction. 


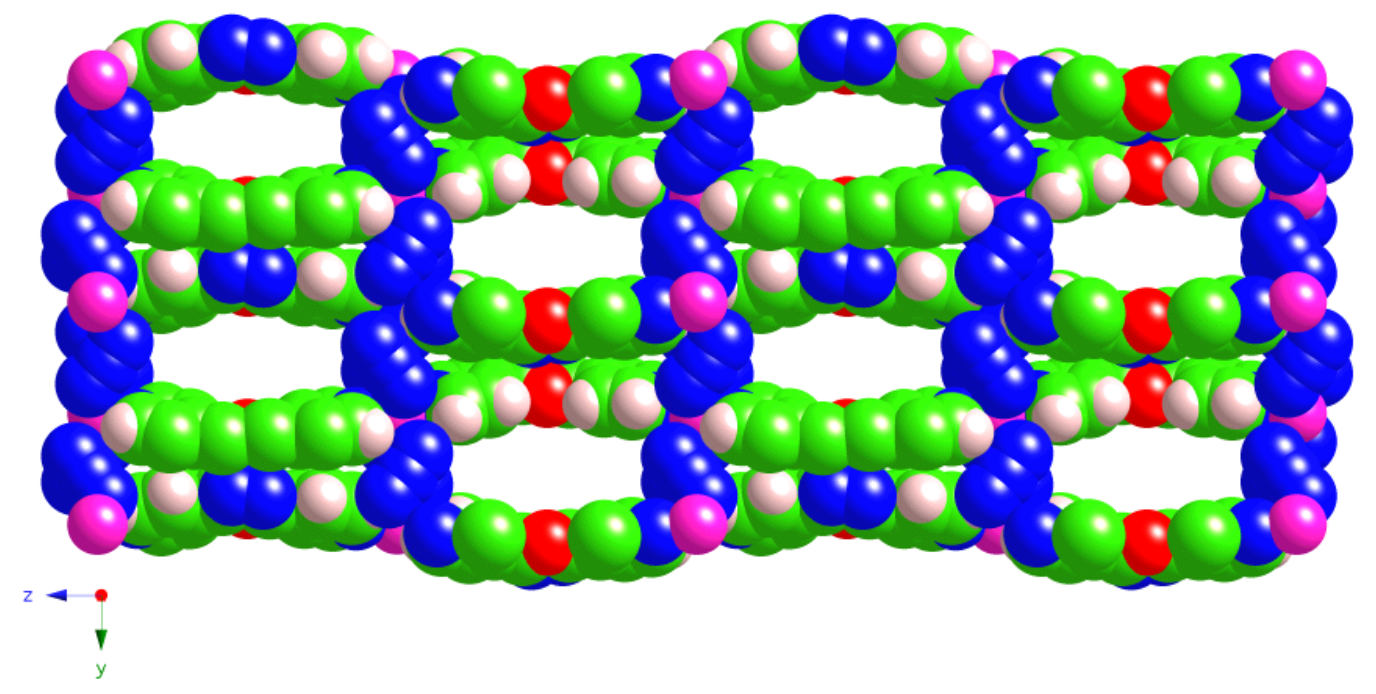

Figure S3. Space-filling model of 2 exhibiting the channels along [100] direction (the voids are occupied by the guest water molecules which are omitted for clarity). 


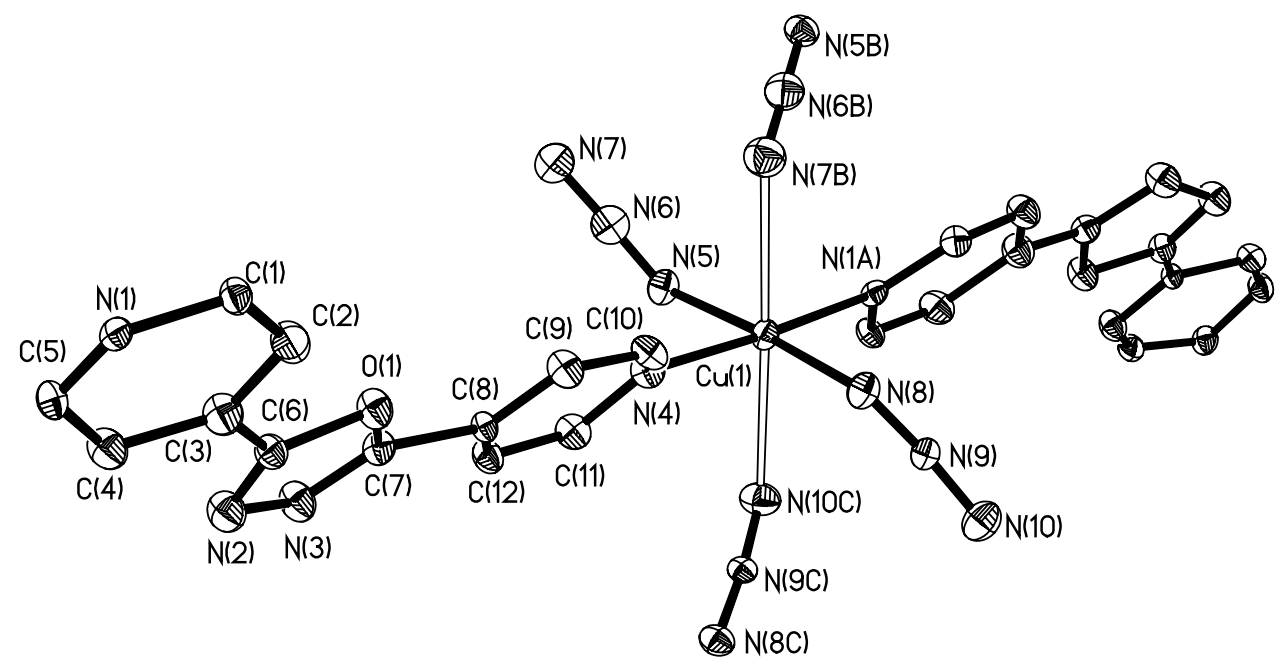

Figure S4. Coordination environment of $\mathrm{Cu}^{\mathrm{II}}$ in complex 2. 


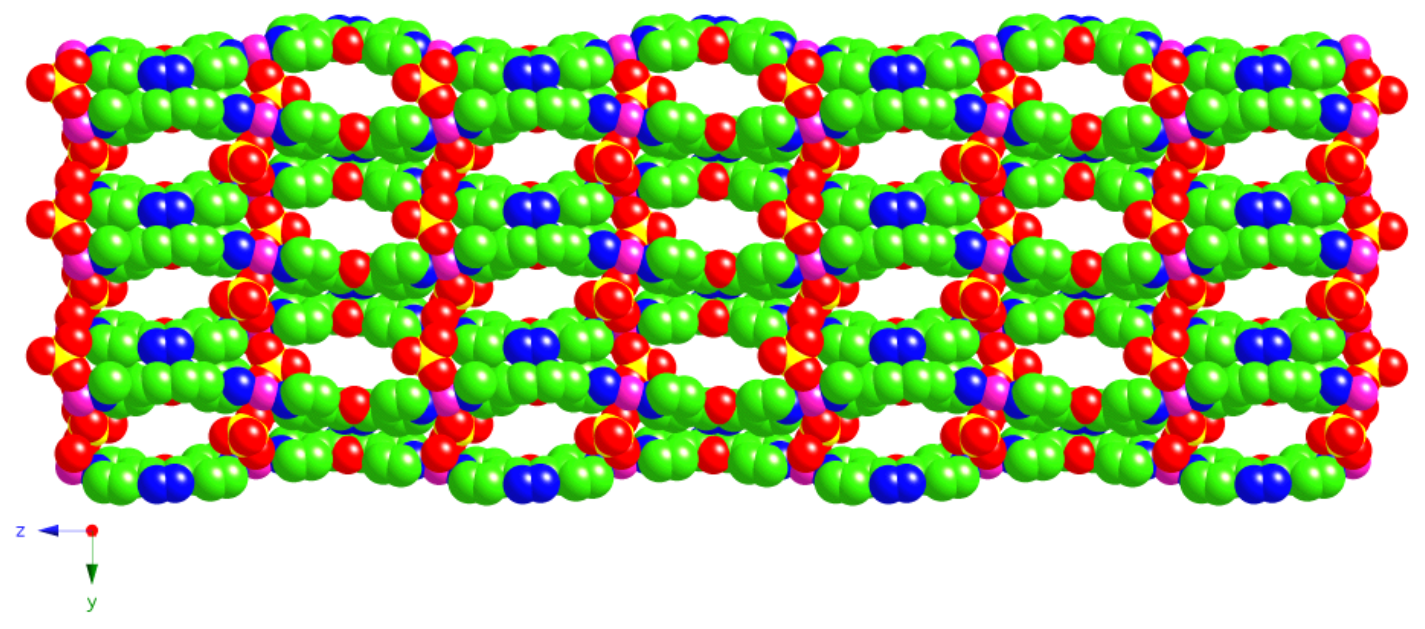

Figure S5. Space-filling model of 3 exhibiting the channels along [100] direction (the voids are occupied by the guest water molecules which are omitted for clarity). 


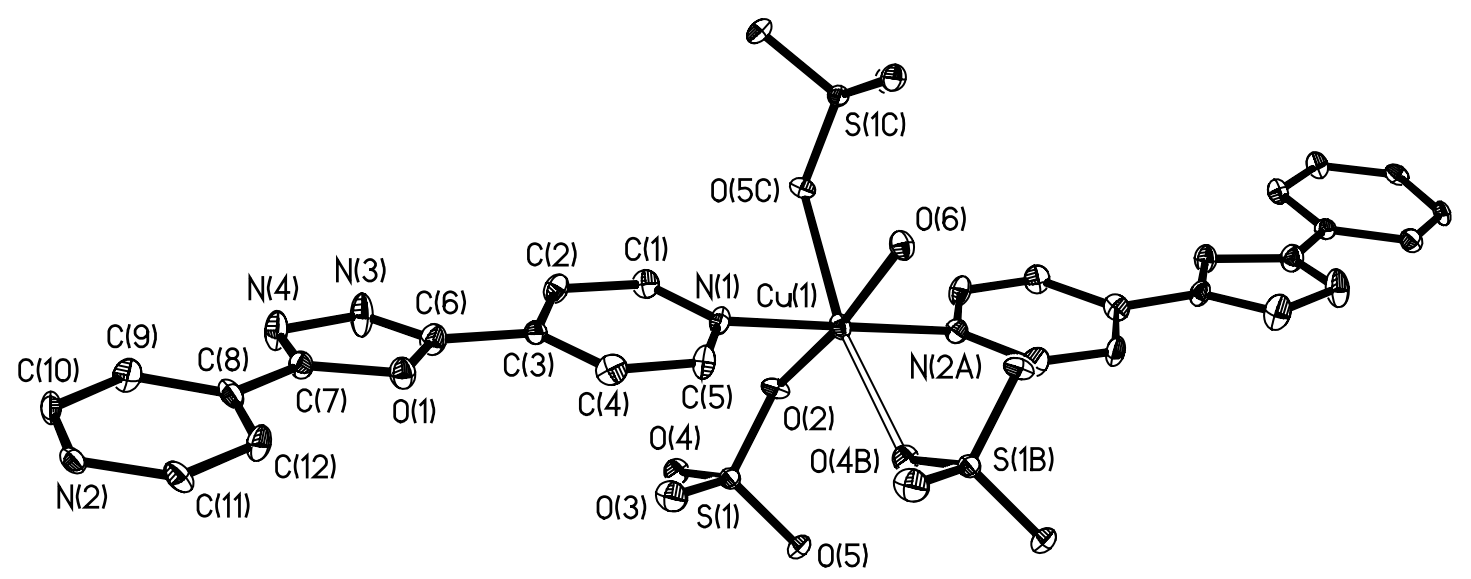

Figure S6. Coordination environment of $\mathrm{Cu}^{\mathrm{II}}$ in complex 3. 


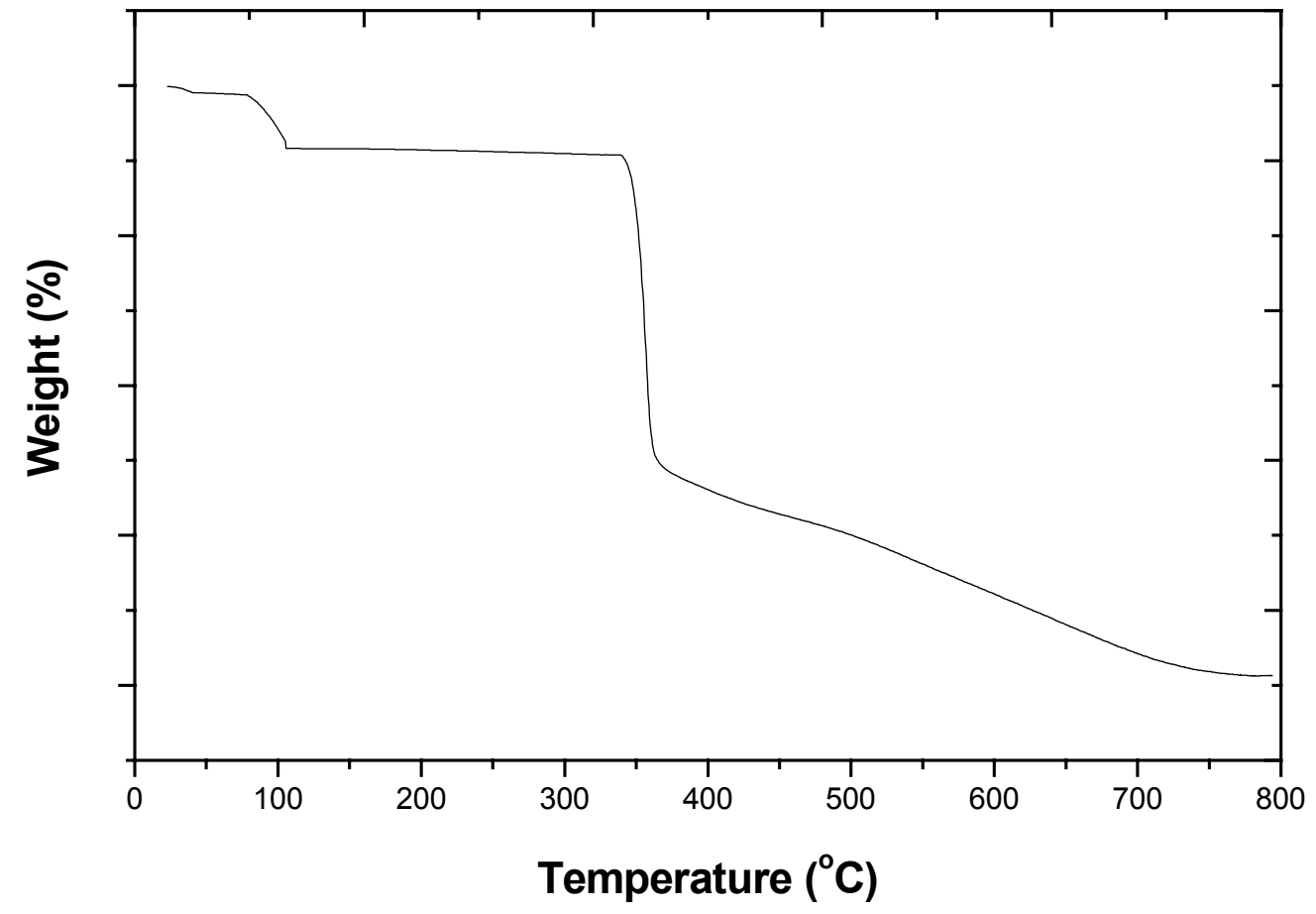

Figure S7. TGA curve of compound 3. 


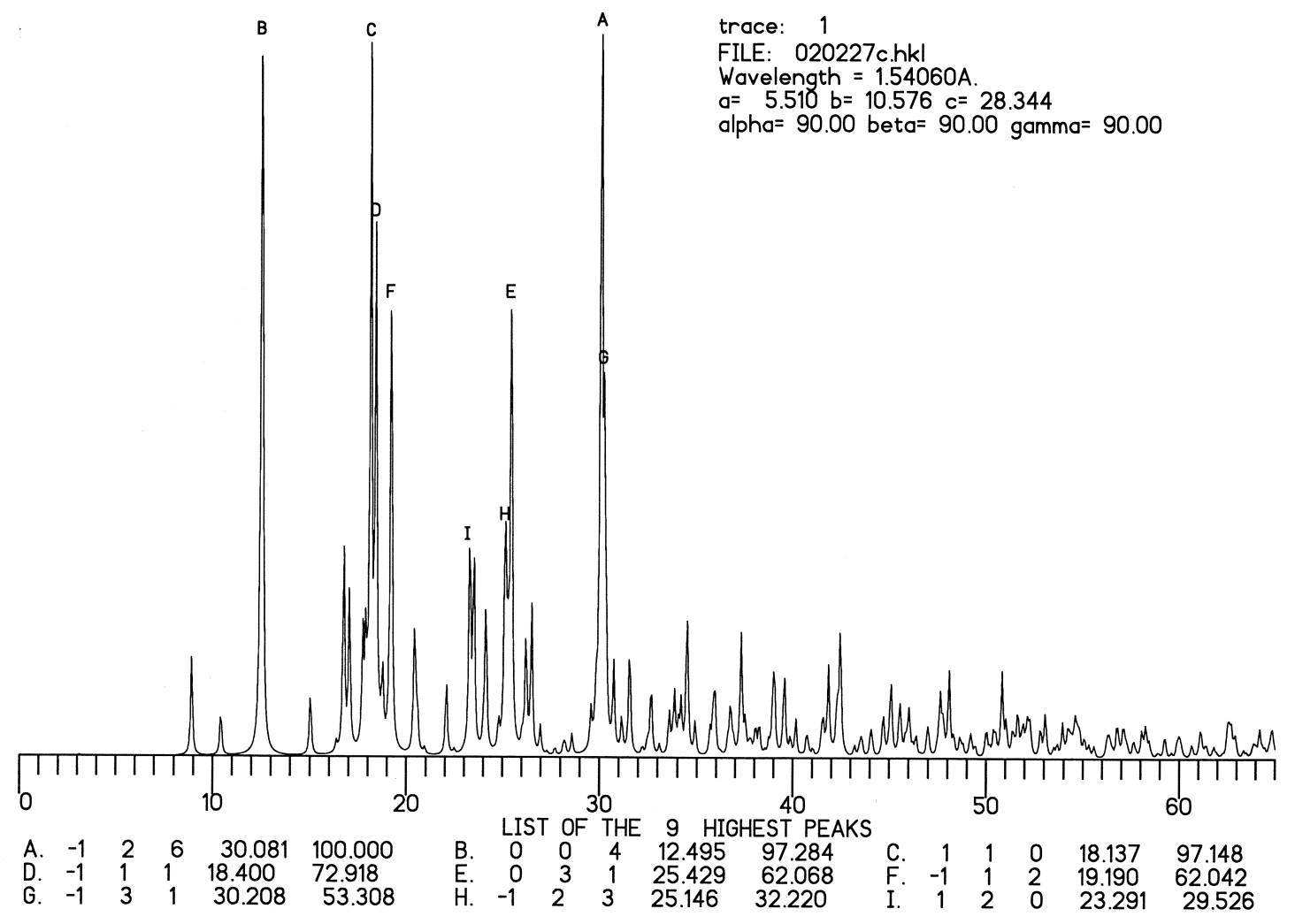

Figure S8. Simulated XRPD pattern for compound 3. 


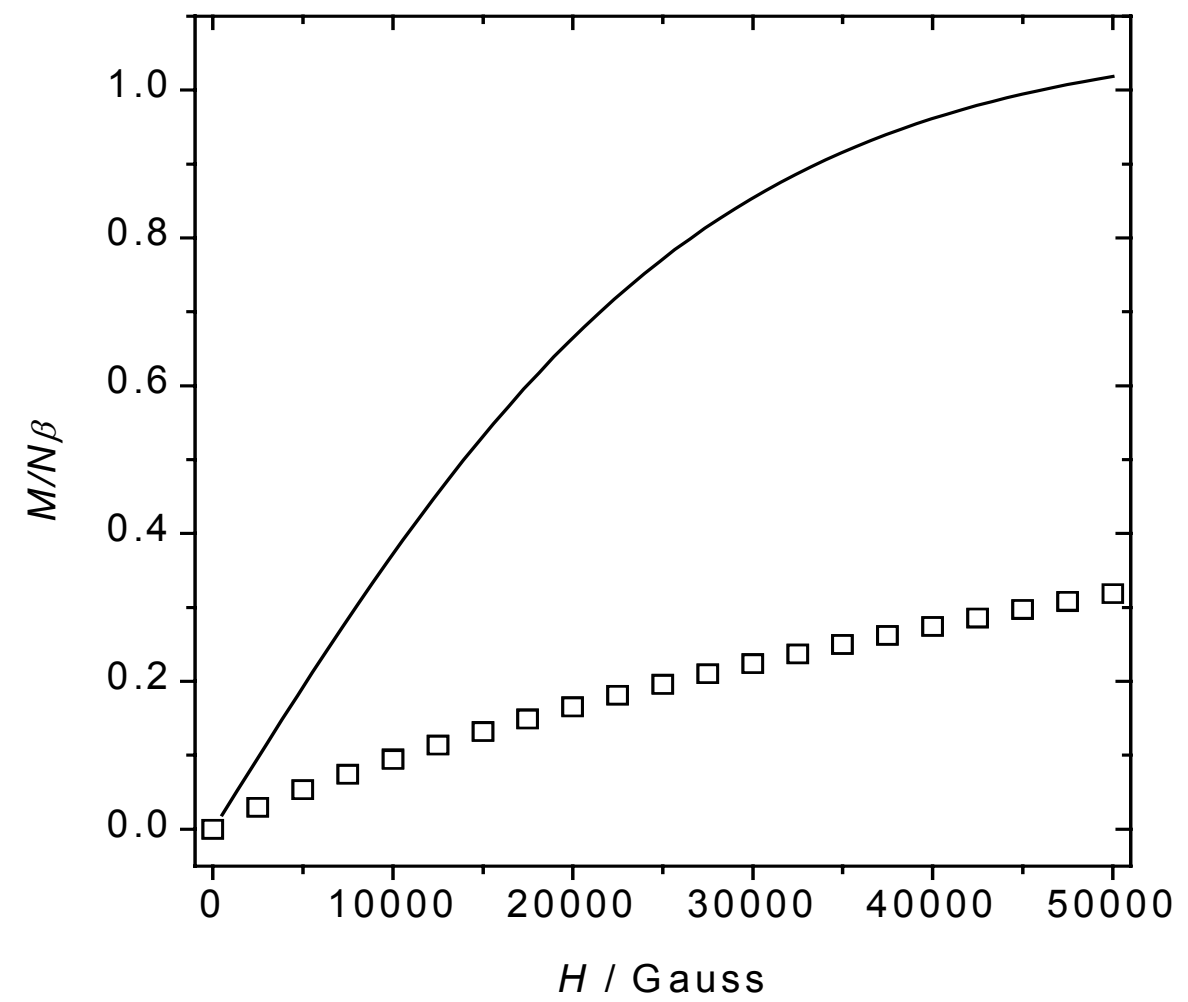

Figure S9. Plot of the $M / N \beta$ (reduced magnetization) for 2 . Open points represent the experimental values and solid line the Brillouin formula for an $S=1 / 2$ ion. 


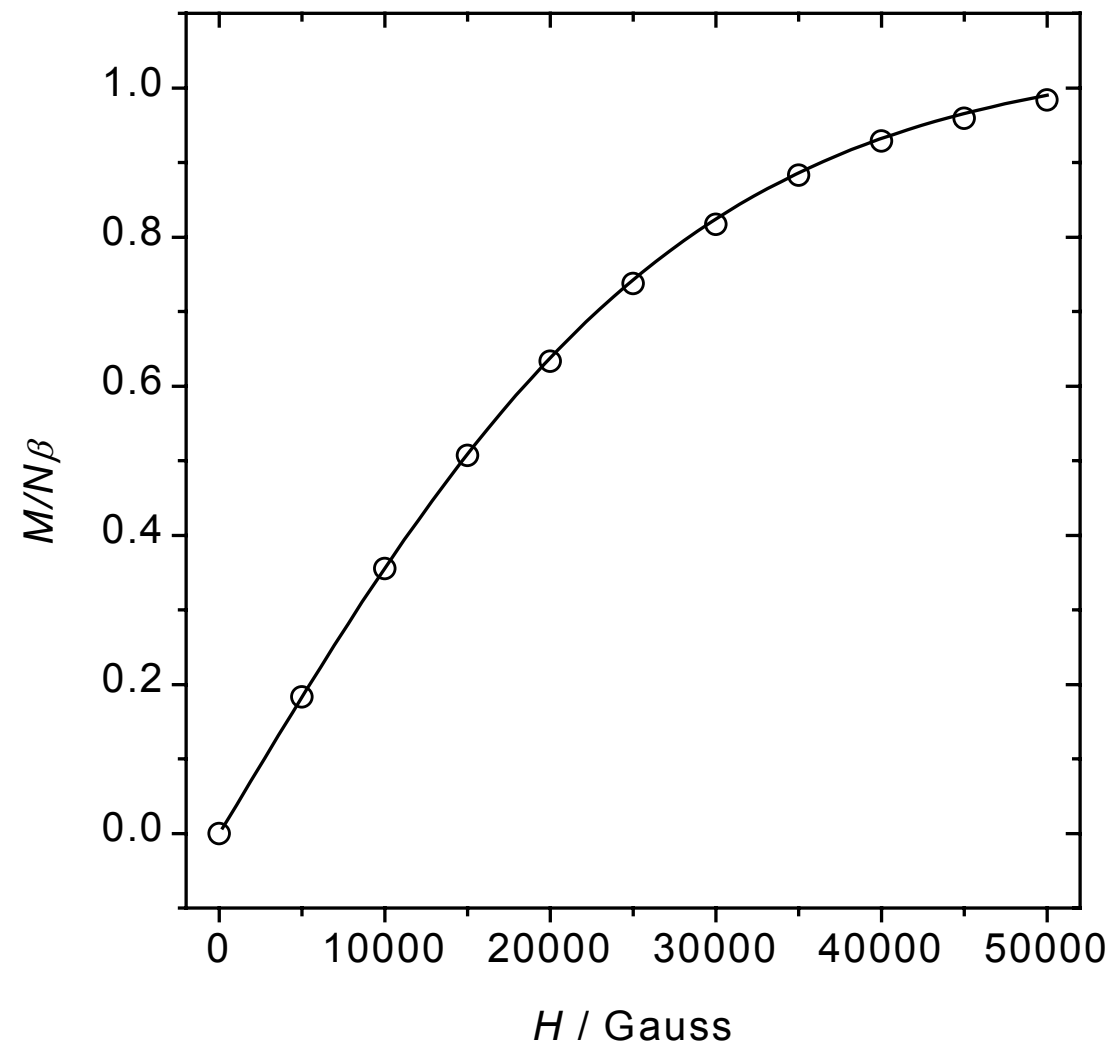

Figure S10. Plot of the $M / N \beta$ (reduced magnetization) for 3. Open points represent the experimental values and solid line the Brillouin formula for an $S=1 / 2$ ion. 